\title{
Las dificultades de aprendizaje de la lectoescritura: implicaciones del diagnóstico de dislexia y la disortografía en Educación Primaria
}

\section{Literacy learning difficulties: implications of dyslexia diagnosis and dysorthography in primary education.}

DOI: $10.46932 / \mathrm{sfjdv2n2-004}$

Received in: January 1st, 2020

Accepted in: March 30th, 2020

\author{
Juani González Muñoz \\ Graduated as an elementaryschool teacher, \\ Institution: RG Formación \\ Calle Rosalía de Castro, 44, 30107 Murcia \\ Belén Cánovas Calderón \\ Graduated as an elementaryschool teacher, \\ Institution: RG Formación \\ Calle Rosalía de Castro, 44, 30107 Murcia \\ Rocío Muñoz Melgar \\ Graduated as an elementaryschool teacher, \\ Institution: RG Formación \\ Calle Rosalía de Castro, 44, 30107 Murcia \\ Jose María Rabal Alonso \\ Professor ISEN Centro Universitario \\ Institution: Universidad de Murcia \\ Calle Campus Universitario, 12, 30100 Murcia \\ E-mail: josemaria.rabal@um.es
}

\section{RESUMEN}

Actualmente las aulas de Educación Primaria están formadas en líneas generales por grupos heterogéneos cuyo alumnado presenta diferencias en cuanto a ritmos de aprendizaje, no todos los niños aprenden igual, ni de la misma manera. Sin embargo, cuando existen dificultades que cumplen con algunos criterios diagnósticos, surgen los llamados trastornos específicos del aprendizaje.

En el caso de la lectoescritura, son muchos los factores que podrían explicar un trastorno específico. Debemos tener en consideración determinados aspectos durante el aprendizaje de la lectura y la escritura para identificar cuáles afectan e interfieren en su rendimiento escolar de manera notable para evaluar bien el porqué de esas dificultades, así como descartar posibles factores que puedan ser la clave del trastorno, tales como una inteligencia límite, un trastorno visual o auditivo etc.

Algunos aspectos a tener en cuenta durante el proceso de aprendizaje lectoescritor de los alumnos pueden ser: la velocidad, precisión y fluidez a la hora de leer y escribir, la comprensión en todas sus dimensiones tales como el conocimiento del significado de las palabras o la comprensión global del texto, así como ideas generales y secundarias. Asimismo, es importante observar el esfuerzo que emplea el alumno y el análisis de los fallos que comete, ya sean ortográficos, de expresión, puntuación o interpretación.

La dislexia es el concepto que utilizamos para englobar a este grupo de síntomas, sin embargo, no existe un único tipo de dislexia y además esta no siempre se presenta de manera aislada, sino que puede combinarse con otras dificultades como el razonamiento matemático o un déficit motriz, por ejemplo.

A continuación, a lo largo del artículo se presenta una explicación de este trastorno, su origen y una 
clasificación según el tipo de dificultad específica que se presente.

Palabras Clave: didáctica, organización escolar, educación inclusiva, necesidades educativas.

\section{ABSTRACT}

Currently, Primary Education classrooms are generally made up of heterogeneous groups whose students present differences in terms of learning rhythms, not all children learn in the same way, nor in the same way. However, when there are difficulties that meet some diagnostic criteria, the so-called specific learning disorders arise.

In the case of literacy, there are many factors that could explain a specific disorder. We must take into consideration certain aspects during the learning of reading and writing to identify which ones affect and interfere in their school performance in a remarkable way to evaluate well the reason for these difficulties, as well as to rule out possible factors that could be the key to the disorder, such as a borderline intelligence, a visual or auditory disorder, etc.

Some aspects to take into account during the process of learning to read and write may be: speed, accuracy and fluency when reading and writing, comprehension in all its dimensions such as knowledge of the meaning of words or global understanding of the text, as well as general and secondary ideas. It is also important to observe the effort used by the student and the analysis of the mistakes he/she makes, whether they are spelling, expression, punctuation or interpretation.

Dyslexia is the concept we use to encompass this group of symptoms, however, there is no single type of dyslexia and it does not always occur in isolation, but can be combined with other difficulties such as mathematical reasoning or a motor deficit, for example.

An explanation of this disorder, its origin and a classification according to the specific type of difficulty is presented throughout the article.

Keywords: didactics, school organization, inclusive education, educational needs.

\section{DIFICULTADES ESPECÍFICAS}

Cuando hablamos de dificultades específicas en el proceso lector, nos referimos a los problemas que surgen a la hora de leer, deletrear o identificar palabras, así como de comprender el texto que se lee.

En contraposición a lo que ocurre con la dislexia adquirida, siendo esta aquella que surge como consecuencia de una lesión cerebral, después de haber alcanzado un nivel lector, estas dificultades se refieren a la dislexia del desarrollo o evolutiva, aquella que se traduce en un déficit inesperado en relación a las otras habilidades cognitivas de la persona y a la adecuada instrucción escolar recibida. En contraste, también pueden darse retrasos no específicos en el aprendizaje de la lectura atribuibles a factores que afectan al rendimiento general, tales como una escolarización tardía o inadecuada o ambiente sociocultural empobrecido.

Lyon, Shaywitz, y Shaywitz (2003), describen la dislexia como una dificultad específica de aprendizaje y cuyo origen es neurobiológico. Los principales signos característicos son el escaso reconocimiento de palabras, la falta de precisión y fluidez en la lectura y problemas con la ortografía y decodificación. Como consecuencia, estos aspectos pueden derivar en la falta de comprensión y la pobreza de vocabulario ya que su experiencia con el lenguaje es escasa.

Estos lectores (lectores diagnosticados de dificultades en el aprendizaje de la lectura — en adelante 
DEAL-) presentan indicios negativos en la lectura fluida y precisa. Asimismo, el patrón que activa las áreas del cerebro dedicadas a procesar el lenguaje, se ve reducido en lectores con dislexia, las técnicas de neuroimagen y estudios en genealogía ponen de manifiesto las diferencias estructurales y funcionales de estas regiones cerebrales. Es por ello, que los genes permiten explicar un 50\% de la varianza, y, en conclusión, la suma de factores ambientales y la proximidad entre familiares con dislexia, así como el grado de parentesco permiten una detección e intervención temprana

El porcentaje de alumnos con este tipo de dislexia en España, se estima en 3,2\% (Jiménez et al., 2009). Afecta concretamente al aprendizaje de la lectura, mientras que la escritura puede ser disgráfica y/o disortográfica.

Son numerosas las nomenclaturas utilizadas para clasificar el tipo de dislexia. En este caso, utilizaremos esta clasificación en base las rutas de procesamiento o acceso al léxico, tal y como las distingue Carmen Silva (2011). De este modo, según la ruta afectada, encontramos la dislexia superficial y la dislexia fonológica, las cuales son descritas a continuación:

Dislexia superficial: en este caso la vía fonológica funciona adecuadamente, pero ocurre lo contrario con la vía léxica y visual, que tiene dificultades para acceder al significado. Como consecuencia de esto ocurre que los niños suelen leer palabras por letras o sílabas, ya que son los fragmentos más pequeños. En este caso, pueden leer las palabras que se escriben y se leen igual (palabras transparentes) y las pseudopalabras, pero no aquellas irregulares y que, por tanto, no coinciden en escritura y pronunciación, estas pueden ser las homófonas como "hola" y "ola, aquellas que presentan fonemas representados por más de una letra como 'b" y 'v" y palabras que llevan la vocal 'u' junto a ' q" y ' g'. Además, tienden a regularizar palabras, así como a cometer fallos de repetición, silabeo o errores de acentuación. Por último, la lectura es lenta.

Dislexia fonológica: aquí ocurre lo contrario, la vía léxica y visual tiene un buen funcionamiento, sin embargo, la dificultad para acceder al significado en este tipo de dislexia se encuentra en la vía fonológica. En este caso la lectura se produce de forma global y aproximada, mediante deducción, permitiendo leer palabras familiares conocidas por su forma, pero no las pseudopalabras o aquellas que desconocen. Se cometen errores muy frecuentes de lexicalización, leyendo por ejemplo "saco" en vez de “'caso", o "'bolo"' en lugar de "'lobo". Además, suelen confundir sufijos de palabras dejándose llevar por aquella que más conocen, por ejemplo, pueden leer 'marino"' en lugar de "'marítimo", estos errores suelen conocerse como errores morfológicos o derivativos. Por último, pueden confundir letras en su lectura si estas se parecen gráficamente, leyendo por ejemplo ''cana' en lugar de 'cara'.

Para terminar con la clasificación recuperada de Carmen Silva (2009), esta termina haciendo hincapié en la dislexia profunda, la cual ocurre cuando ambas vías están afectadas y por tanto, pueden cometer numerosos fallos de cualquier tipo. 


\section{DIFICULTADES DE COMPRENSIÓN}

En este caso, al contrario que las dificultades específicas anteriormente mencionadas de origen neurobiológico, las dificultades de comprensión tienen origen cognitivo. Suelen presentarse en alumnos con bajo nivel atencional o comprensivo, dificultando específicamente al acceso del significado.

Algunos aspectos que pueden influir en el inadecuado uso de las estrategias cognitivas y metacognitivas y, por tanto, su funcionamiento, pueden ser la inexistencia de relación entre las ideas del texto como un todo coherente y lleno de significado, así como la ausencia de integración de sus conocimientos previos con los nuevos. Debido a lo anteriormente descrito, los alumnos leen palabra a palabra sin relacionar ideas, obteniendo un listado escaso de las mismas sin significado ni sentido. Esto conlleva a la falta de realización de inferencias por parte del alumnado, y las inferencias y el acto de deducir suponen la base de la comprensión. Oakhill (1984) puso de manifiesto que los lectores con dificultades de comprensión, contestaban adecuadamente a las preguntas cuyas respuestas aparecían en el texto de manera literal, sin embargo, no podían hacer lo mismo con aquellas en las que debían realizar inferencias aun con el texto recién leído.

Para concluir con las dificultades de comprensión, podemos decir que la falta de habilidades comprensivas, se refleja en lectores que desconocen el objetivo de la lectura, no planifican sus estrategias metacognitivas para enfrentarse al acto de leer y no llevan a cabo una supervisión de lo leído, es decir, no realizan una segunda lectura o no se apoyan en contextos familiares o significativos para llegar a su comprensión.

\subsection{DIFICULTADES DE APRENDIZAJE EN LOS PROCESOS DE ESCRITURA.}

Los niños con dislexia suelen presentar dificultades en la escritura, puesto que este proceso es más complejo y demanda más implicación que el lector. La disgrafía evolutiva, como ocurre en la dislexia, es un trastorno que suele iniciarse en la infancia y supone un déficit en el proceso escritor, tales como la ortografía, la gramática o la semántica. Jiménez et al. (2009) determinaron que el porcentaje de alumnos con dificultades de aprendizaje de la escritura (en adelante DAE) era de un 5,4\%. Este tipo de disgrafía no tiene nada que ver con aquella que se explica por una lesión cerebral una vez instaurada la escritura. Asimismo, existen diferentes retrasos en el aprendizaje lector que se explican por variables ajenas al sujeto tales como una escolarización tardía o ausencia de escolarización, desconocimiento del idioma o ambiente familiar empobrecido, entre otras. Es por ello importante, discriminar entre el desconocimiento o empleo incorrecto de los procesos cognitivos o motóricos y la verdadera DAE.

En este caso, origen de la disgrafía evolutiva lo encontramos en los procesos que se activan a la hora de escribir, pudiendo ser estos mismos cognitivos (procesos internos) o motóricos (procesos externos), dando así lugar a dos tipos de disgrafía: disgrafía central (cognitiva) y disgrafía periférica (motórica). 


\section{DISGRAFÍA CENTRAL}

Dentro de esta tipología, encontramos variaciones, sin embargo, en todas ellas los procesos afectados son los internos, pudiendo ser estos mismos léxicos básicos o complejos.

\subsection{DISGRAFÍA CENTRAL EN LOS PROCESOS BÁSICOS LÉXICOS.}

Disgrafía central fonológica (disortografía natural): este tipo de disgrafía recibe el daño en la vía fonológica (indirecta) y por tanto apoya su escritura en la léxica (visual o directa). Aquí se producen fallos de omisión de grafemas, así como sustituciones, adiciones o inversiones, debido a la dificultad de transformar el sonido (fonema) en grafema. Ejemplo de disortografía natural:

\section{DISORTOGRAFÍA NATURAL}

Caba E bías dedo nu patibo de cocolate. Meloto mo con un torzo be dizcoco y galeta. Bes pues fw la qarpue con mi pero, nos lo paso en garden. Puero pue lobos tos bías sean azí.

Disgrafía central superficial (disortografía arbitraria): aquí el déficit se encuentra en la vía léxica (visual o directa) y por tanto la escritura se apoya en la vía fonológica, que tiene un buen funcionamiento. En este tipo de disgrafía encontramos las llamadas faltas de ortografía de palabras irregulares o poligráficas. Sin embargo, aquellas palabras regulares, es decir, que se escriben tal y como se pronuncian, se escriben correctamente al sustentar el proceso escritor en la vía fonológica, no obstante, pueden producirse errores de automatización de las imágenes de las palabras, sobre todo aquellas que suenan igual, por ejemplo, "vaca" o 'baca'. Ejemplo de disortografía arbitraria:

\section{DISORTOGRAFÍA ARBITRARIA}

el hotro dia fui al campo hay vi muchas bacas. torros. gayinas y un espanta pagaros. fui con el colegio y ivamos mi profesora mi amigo hantonio y mi amiga maria. cuando bolbimos a casa le conte a mi madre todo lo ke havia visto. aora es ella la ke kiere hir.

Disgrafía central profunda (disortografía mixta): en este caso, el déficit se encuentra tanto en la vía fonológica como en la léxica, viéndose ambas rutas afectadas. Por ello, los errores producidos pueden ser de todo tipo, tanto de ortografía natural como arbitraria, sin embargo, la vía fonológica suele ser la más mecanizada, tienden a apoyarse en ella para solucionar sus fallos y potencian así los errores de ortografía arbitraria. Cuando se atiende a este tipo de disgrafía central, debe ser muy importante el equilibrio y la coordinación del refuerzo en ambas vías. 


\subsection{DISGRAFÍA CENTRAL EN LOS PROCESOS COMPLEJOS.}

Este tipo de disgrafía presenta obstáculos a la hora de llevar a cabo la elaboración de un texto. De este modo, los procesos básicos de composición de textos se ven afectados. En primer lugar, no realizan una planificación de lo que van escribir, no tienen en cuenta a los emisores a los que se dirigen y existe una ausencia de objetivos principales del texto. Además, las ideas no presentan una jerarquía, sino que se presentan desordenadas. En segundo lugar, a la hora de producir el texto, se cometen errores morfosintácticos, no existen nexos que unan las ideas y existe una ausencia de concordancia entre las oraciones y palabras. Por último, no realizan una revisión del texto que les permita comprobar si se han cumplido los objetivos.

\section{DISGRAFÍA PERIFÉRICA}

Este tipo de disgrafía corresponde con el déficit, como decíamos al principio, de los procesos externos o motóricos del sujeto. En este caso, la caligrafía se ve notablemente afectada por no seguir un patrón adecuado de escritura, dando lugar a letra de tamaño desigual y desproporcionada y grafismo ilegible. Asimismo, suelen mezclar mayúsculas y minúsculas y distintos tipos de letra. Por último, en cuanto al aspecto externo y estético del texto, este suele presentar líneas inclinadas, frases desordenadas, márgenes desiguales o párrafos desorganizados. Ejemplos de disgrafía periférica:

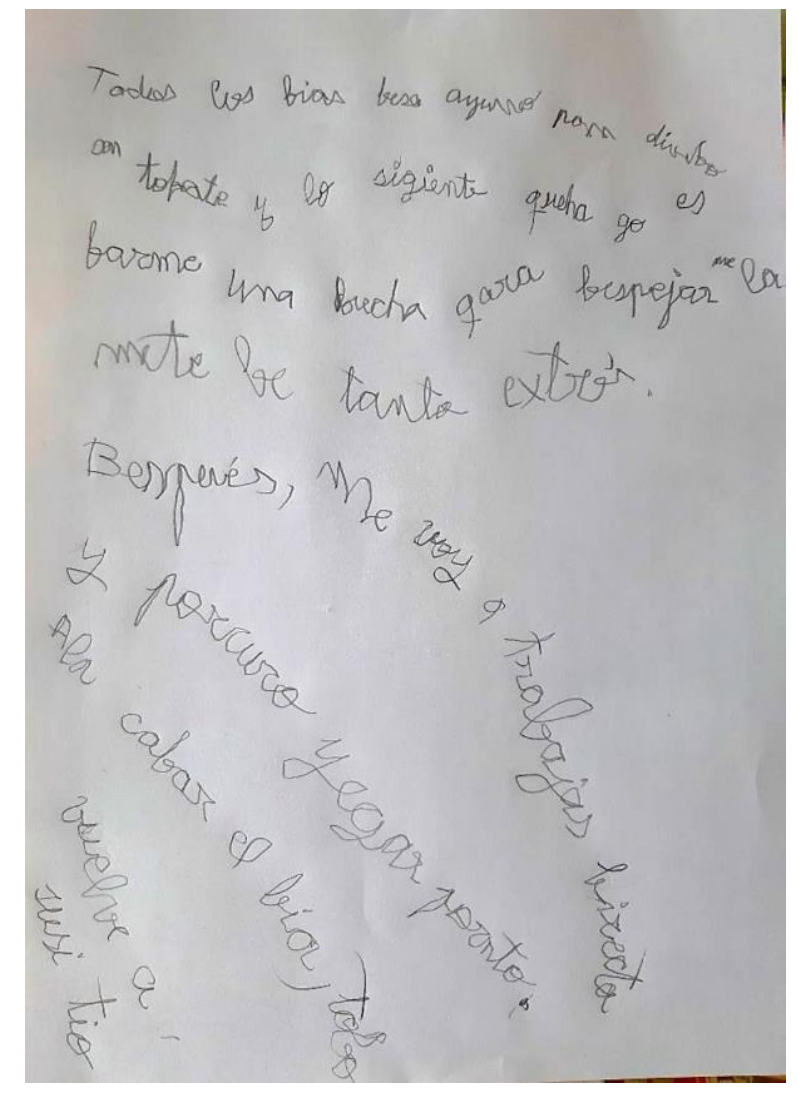




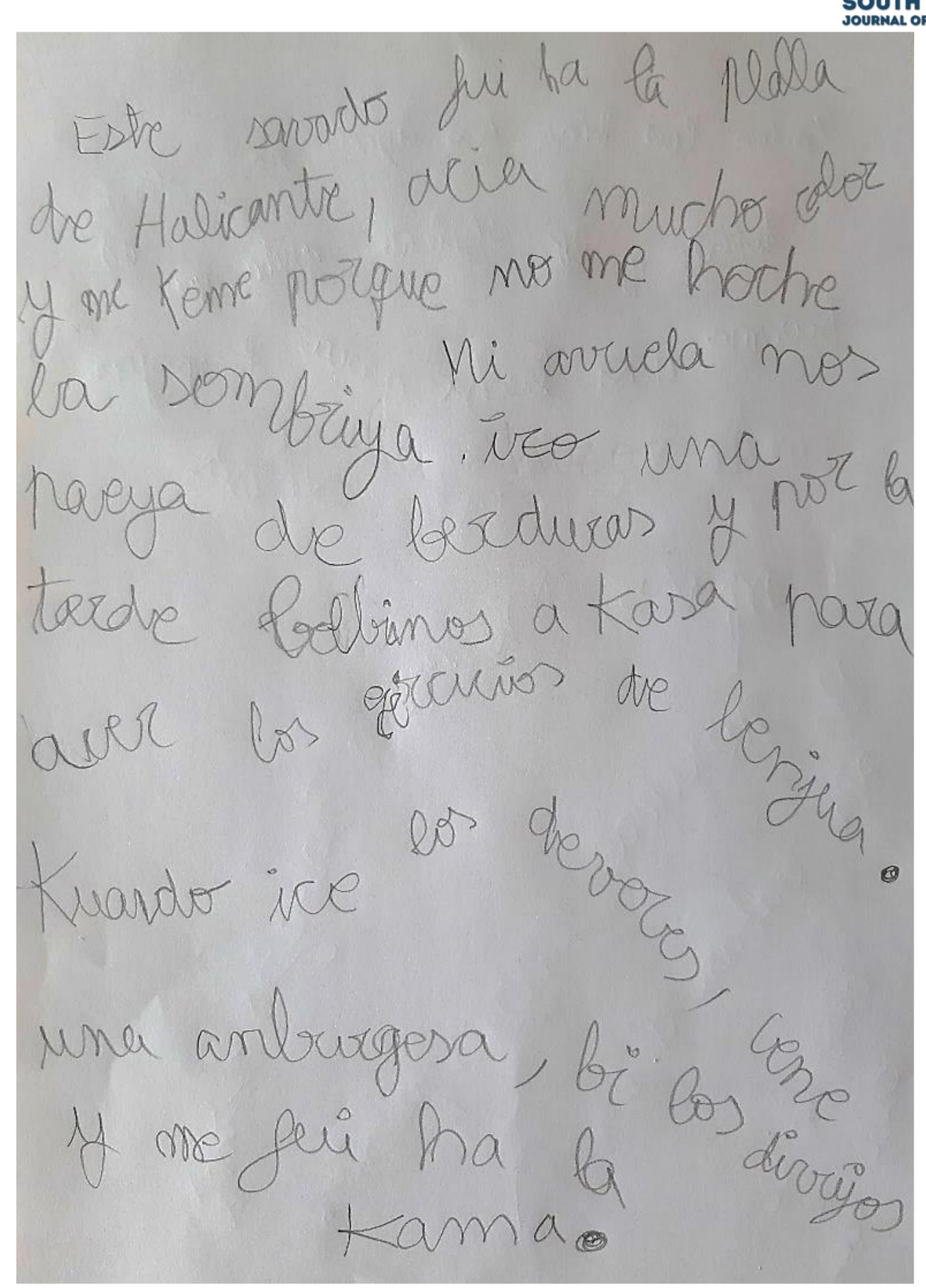

\section{CONCLUSIÓN}

En los trastornos específicos del lenguaje podemos ver diferentes niveles de gravedad. En el caso de la lectoescritura es importante conocer estas dificultades, saber identificarlas en edades tempranas y durante el proceso de aprendizaje para no llegar a la automatización de conductas erróneas que podrían dificultar el proceso y agudizarse en la vida adulta.

Por otra parte, la dislexia no es un factor que determine el nivel de inteligencia de una persona, ya que hay personas disléxicas verdaderamente inteligentes. Sin embargo, los alumnos que presentan trastornos específicos tienen la necesidad en la mayoría de ocasiones de recibir adaptaciones en actividades 
relacionadas con la escritura y la lectura que faciliten su inclusión y aprendizaje en el aula.

Por último, es importante manejar de manera adecuada la autoestima de las personas con dislexia, ya que esta puede verse dañada y elevar los niveles de frustración. Es por ello necesaria, la coordinación de profesionales de la educación a la hora de emplear recursos y dotar de herramientas a quienes presenten este tipo de dificultades.

\section{PROPUESTA DE ACTIVIDADES:}

- Señala la silaba correcta en cada caso e inventa una nueva palabra:

\begin{tabular}{|c|c|c|c|c|}
\hline Palabra: & $\begin{array}{c}\text { Primera } \\
\text { sílaba }\end{array}$ & Segunda sílaba & Tercera sílaba & $\begin{array}{c}\text { Inventa otra palabra que } \\
\text { contenga esa sílaba }\end{array}$ \\
\hline $\mathrm{Ca}-\mathrm{X}-\mathrm{za}$ & to & be & $\mathrm{cu}$ & Abeto \\
\hline Es $-X-b a$ & co & lo & bu & \\
\hline Es $-\mathrm{pa}-$ & fe & $\mathrm{ca}$ & $\mathrm{da}$ & \\
\hline Col-me - X & $\mathrm{da}$ & $\mathrm{ba}$ & na & \\
\hline Pro - yec - & bo & co & to & \\
\hline Es $-X-$ so & be & pe & co & \\
\hline $\mathrm{Cu}-\mathrm{X}-1 \mathrm{lo}$ & chi & $1 \mathrm{li}$ & $\tilde{n} \mathrm{i}$ & \\
\hline $\mathrm{X}$ - bli - co & $\mathrm{pu}$ & po & $\mathrm{pa}$ & \\
\hline$X$ - ne -la & $\mathrm{ga}$ & $\mathrm{ca}$ & tu & \\
\hline $\mathrm{Fa}-\mathrm{X}-\mathrm{lia}$ & li & lo & $\mathrm{mi}$ & \\
\hline Tor $-\mathrm{X}$ - do & ci & di & ce & \\
\hline $\mathrm{Fu}-\mathrm{tu}-\mathrm{X}$ & $\mathrm{ra}$ & ro & $\mathrm{ru}$ & \\
\hline
\end{tabular}

- Ordena las sílabas y forma una nueva palabra utilizando la sílaba que aparece coloreada.

\begin{tabular}{|l|l|l|l|l|l|}
\hline Primera sílaba & $\begin{array}{l}\text { Segunda } \\
\text { sílaba }\end{array}$ & Tercera sílaba & Cuarta sílaba & $\begin{array}{l}\text { Palabra } \\
\text { obtenida }\end{array}$ & $\begin{array}{l}\text { Palabra } \\
\text { inventada }\end{array}$ \\
\hline le & a & a & grí & alegría & Camaleón \\
\hline Dis & pli & ci & na & & \\
\hline ce & la & pi & ro & & \\
\hline man & na & da & ri & & \\
\hline ma & po & ri & sa & & \\
\hline ta & pan & nes & lo & & \\
\hline e & le & te & fan & & \\
\hline le & vi & te & sión & & \\
\hline li & gra & bo & fo & & \\
\hline za & ba & la & ca & & \\
\hline to & fa & vo & ri & & \\
\hline la & cu & li & pe & & \\
\hline
\end{tabular}

- Coloca una X según las sílabas que contenga cada palabra. Después escribe otra palabra diferente que tenga las mismas sílabas. 


\begin{tabular}{|l|c|c|c|c|c|c|c|c|c|}
\hline \multirow{2}{*}{ Palabra } & \multicolumn{7}{|c|}{ ¿Cuántas sílabas tiene? } & \multirow{2}{*}{$\begin{array}{c}\text { ¿Conoces alguna otra } \\
\text { palabra con el mismo } \\
\text { número de silabas? }\end{array}$} \\
\cline { 2 - 10 } & 1 & 2 & 3 & 4 & 5 & 6 & 7 & 8 & \\
\hline Ornitorrinco & & & & & $\mathrm{X}$ & & & & Extraordinario \\
\hline Emperador & & & & & & & & & \\
\hline Cuadrado & & & & & & & & & \\
\hline Libreta & & & & & & & & & \\
\hline Sol & & & & & & & & \\
\hline Ratón & & & & & & & & \\
\hline Originalidad & & & & & & & & & \\
\hline Irresponsabilidad & & & & & & & & & \\
\hline Marco & & & & & & & & & \\
\hline Proyecto & & & & & & & & & \\
\hline Mar
\end{tabular}

- Estas palabras se han dado la vuelta y están desordenadas. ¿Podrías ordenarlas?

\begin{tabular}{|c|l|}
\hline NOBAJ & \\
\hline ORDAUC & \\
\hline ETNOZIROH & \\
\hline ASAC & \\
\hline OLUGNAIRT & \\
\hline EHCON & \\
\hline ATERBIL & \\
\hline OTXET & \\
\hline SENORRACAM & \\
\hline RODALLINROTSED & \\
\hline ADREIUQZI & \\
\hline LOS & \\
\hline OREBMOB & \\
\hline ASOPIRAM &
\end{tabular}




\section{REFERENCIAS}

Alonso, J. M. R., \& Romero, M. G. (2018). Disortografía. In IV Congreso Virtual Internacional sobre Innovación Pedagógica y Praxis Educativa INNOVAGOGÍA 2018: libro de actas: 20, 21 y 22 de marzo 2018 [resúmenes] (p. 301). AFOE. Asociación para la Formación, el Ocio y el Empleo.

Jiménez, J. E., Guzmán, R., Rodríguez, C., \&Artiles, C. (2009). Prevalencia de las dificultades específicas de aprendizaje: la dislexia en español. Anales de Psicología/Annals of Psychology, 25(1), 78-85.

Lyon, G.R., Shaywitz, S. \&Shaywitz, B.A. (2003). A definition of dyslexia. Annals of Dyslexia. 53, 1-14. Oakhill, J. (1984). Inferential and memoryskills in children'scomprehension of stories. British Journal of EducationalPsychology, 54, 31-39.

Romero, M. G., Alonso, J. M. R., \& Romero, J. G. (2020). Aproximación conceptual a la dislexia en las aulas de Educación primaria. Brazilian Journal of Development, 6(4), 20157-20165.

Romero, M. G., Alonso, J. M. R., \& Romero, J. G. (2020). Aproximación conceptual a la disortografía. Aportaciones docentes. Brazilian Journal of Development, 6(4), 18814-18820.

Silva, Carmen. (2011). Tipos de dislexia. Portal de información sobre dislexia Ladislexia.net. Recuperado de: http://www.ladislexia.net/tipos-clasificacion/ 